DOI:10.2478/rrlm-2021-0010

\title{
The positive rate of IgM and IgG antibodies against SARS-CoV-2 is similar in severe and non-severe COVID-19 patients
}

\author{
Nan Jiang ${ }^{1 \#}$, Yaoyao Sun ${ }^{1,7 \#}$, Hongyan Sun ${ }^{2 \#}$, Bo Yang ${ }^{3}$, \\ Juan $\mathrm{Tan}^{4}$, Huan $\mathrm{Sun}^{5}$, Shuai Wang ${ }^{6}$, Qiong $\mathrm{Yu}^{7 *}$
}

1. Department of Emergency, China-Japan Union Hospital of Jilin University, China

2. Department of biobank, China-Japan Union Hospital of Jilin University, China

3. Institute of Organ Transplantation, Tongji Hospital, Tongji Medical College, Huazhong University of

Science and Technology, China

4. Department of anesthesiology, Tongji hospital of Tongji Medical college, Huazhong University of science and Technology, China

5. Department of Cardiology, China-Japan Union Hospital of Jilin University, China

6. Department of Critical Care Unit, China-Japan Union Hospital of Jilin University, China

7. Department of Epidemiology and Biostatistics, School of Public Health Jilin university, China

\begin{abstract}
Background: Coronavirus disease 2019 (COVID-19) has spread rapidly in China and globally. In order to control the spread of the epidemic, it is important to find an efficient diagnostic method. Objectives: The aim of this study was to assess the responses of antibodies during SARS-CoV-2 infection in relation to disease severity and to evaluate the association between the positive rate of antibody detection and nucleic acid test. Methods: Ninety patients with SARS-CoV-2 infection were recruited in this retrospective observational study. Demographic, clinical data, and SARS-CoV-2 IgM and IgG antibodies in serum specimens were detected at 4 and 6 weeks after diagnosis. Results: IgM and IgG antibody levels showed a decreased tendency, the titers at week 4 were higher than the titers at week 6: The positive rates of IgM at week 4 and 6 were $92.9 \%$ and $67.9 \%$, respectively. The positive rates of $\operatorname{IgG}$ at week 4 and week 6 were $100 \%$. No association was found between the positive rate of antibody detection at week 4 or 6 and that of nucleic acid test $(P>0.05)$. No difference between the positive rate of antibodies against SARS-CoV-2 in severe and non-severe COVID-19 patients was observed. Conclusions: Antibody detection is an effective means in the diagnosis of COVID-19. The titer and positive rate of IgM are lower than those of IgG in the first six weeks after infection. Positive rate of antibodies was not different between severe and non-severe patients.
\end{abstract}

* Corresponding author: Qiong Yu, Department of Epidemiology and Biostatistics, School of Public Health Jilin university, 1163 Xinmin Street Chaoyang District, 130021, Changchun, China. E-mail: yuqiong@jlu.edu.cn

" Authors with equal contribution to this work. 
Keywords: COVID-19, SARS-CoV-2, Serological test

Received: $1^{\text {st }}$ October 2020; Accepted: $4^{\text {th }}$ January 2021; Published: $15^{\text {st }}$ January 2021

\section{Background}

Coronavirus disease 2019 (COVID-19) caused by the severe acute respiratory syndrome coronavirus 2 (SARS-CoV-2) has spread rapidly in China and globally. The global mortality rate was reported to range from $1.5-3.6 \%$ (1). The number of infections and deaths is still increasing daily, such widespread outbreaks lead to serious public health threats and tremendous economic loss. COVID-19 has the characteristics of rapid transmission, atypical clinical symptoms, and it is easy to be misdiagnosed. In order to control the spread of the epidemic, it is important to find an efficient diagnostic method.

The standard diagnosis of COVID-19 infection depends mainly on viral antigen detection through real-time reverse transcription polymerase chain reaction (RT-PCR) assays of nasal swab, pharyngeal swab, and other specimens (2). However, numerous "suspected" COVID-19 cases with typical clinical characteristics and/ or specific computed tomography (CT) imaging have not been diagnosed. Nucleic acid test carries the obvious risk of false-negatives due to low viral loads in specimens, sampling site, transportation and storage, laboratory testing conditions and personnel operations. Additionally, many factors such as insufficient kits, disease course, and patients' immune status can influence the diagnostic process, which limit the use of this method for outbreak control. Compared with RT-PCR, the antibody detection is usually faster, cheaper, and easier to operate. Titers of SARSCoV-2 antibodies can provide information on the progress of viral infection. SARS-CoV-2 has some similarities to SARS-CoV and MERS$\mathrm{CoV}$ as a beta-coronavirus. IgM is detectable as early as 3-6 days after infection, and IgG can be detected after 8 days (3). Rapid and specific an- tibody detection can help to confirm or exclude suspected cases and has been recommended by the Guidelines of Diagnosis and Treatment for COVID-19 (Seventh Trial Version) issued by National Health Commission of China (4).

The aim of this study was to assess the responses of antibodies during SARS-CoV-2 infection and evaluate the association between the positive rate of antibody detection and nucleic acid test.

\section{Methods}

\section{Patients}

This retrospective observational study was conducted at the Tongji Hospital, Tongji Medical College, Huazhong University of Science and Technology from February 2, 2020 to March 25,2020 . Ninety patients with SARS-CoV-2 infection were recruited in this study according to the Guidelines of Diagnosis and Treatment for COVID-19 (Seventh Trial Version) of China. Confirmed cases should meet one of the following criteria: (1) a positive result of SARS-Cov-2 RNA was detected by RT-PCR, (2) the virus gene sequencing of blood samples or respiratory was highly homologous with SARS-CoV-2, and (3) SARS-CoV-2 IgM and IgG antibodies in serum specimens were positive; IgG antibodies changed from negative to positive or increased 4 times or more in the recovery phase compared with the acute phase. The severity of COVID-19 should meet one of the following criteria: (1) respiratory rate $\geq 30 / \mathrm{min}$, (2) finger of oxygen saturation $\leq 93 \%$ in a resting state, and (3) arterial oxygen tension /inspiratory oxygen fraction $\leq 300 \mathrm{~mm}$ Hg. Clinical retrospective data including demographic characteristics, clinical symptoms and laboratory findings were retrieved from medical records. The study was approved by the Ethical Committee of Tongji Hospital, Tongji Medical 
College, Huazhong University of Science and Technology and China-Japan Union Hospital of Jilin University. Informed consent was obtained from all subjects.

\section{SARS-CoV-2 antibody detection}

The new coronavirus (2019-nCoV) IgM/IgG antibody detection kit (colloidal gold method) was provided by Vazyme Biotech Co., Ltd., Nanjing, China and the storage temperature was $4^{\circ} \mathrm{C}$. Antibody detection adopts capture method and solid-phase chromatographic immunoassay for detection. The sensitivity was $91.54 \%(95 \% \mathrm{CI}$ : $86.87 \%, 94.65 \%)$, and the specificity was 97.02\% (95\%CI: 94.74\%, 98.33\%). IgM and IgG titers $\leq 10 \mathrm{AU} / \mathrm{ml}$ were considered as negative, and $>10 \mathrm{AU} / \mathrm{ml}$ were classified as positive.

\section{Statistical analysis}

Continuous variables are described as the mean \pm standard deviation (SD) or the median (interquartile range, IQR). Categorical variables were presented as the frequency and percentage (\%) and assessed using Pearson Chi-square test or Fisher's exact test. Wilcoxon matched-pairs signed-ranks test was used to compare the antibody titers at week 4 and 6. McNemar's test was used to analyze the difference in the positive rate of antibody between $\operatorname{IgG}$ and $\operatorname{IgM}$ at week 4. Results with two-sided $P$ values less than 0.05 were deemed to be statistically significant. All analyses were performed by IBM SPSS 24.0.

\section{Results}

A total of 90 patients with confirmed COVID-19 were included in this study, 40 (44.4\%) male patients and $50(55.6 \%)$ female ones. The median age was 65.00 years old (interquartile range: 55.00-72.25 years). Sixteen (17.8\%) patients were classified as severe COVID-19 and 71 (78.9\%) patients were considered as non-severe COVID-19. Fever, cough, dyspnea, headache, and diarrhea were common symptoms. Table 1 shows the baseline characteristics of patients with COVID-19. Titers of SARS-CoV-2 antibodies were tested twice. Ninety patients were conducted antibody detection at week 4 , however, we tested 84 patients at week 6 because six patients were discharged from hospital. IgM titers at week 4 and 6 were $60.50 \mathrm{AU} / \mathrm{ml}$ and 17.50 $\mathrm{AU} / \mathrm{ml}$, respectively, whereas IgG titers at week 4 and 6 were $189.00 \mathrm{AU} / \mathrm{ml}$ and $108.00 \mathrm{AU} /$ $\mathrm{ml}$, respectively. IgM and $\operatorname{IgG}$ antibody levels showed a decreased tendency, the titers at week 4 were higher than the titers at week 6 (figure $1)$. The differences were statistically significant $(P<0.001)$ (Table 2).

We found that most patients had higher levels of $\operatorname{IgM}$ and $\operatorname{IgG}$ at week 4 than week 6. Table 3 show the results of comparison of the posi-

Table 1. The clinical characteristics and biochemical values of patients with COVID-19 $(\mathrm{N}=90)$

\begin{tabular}{lc}
\hline Characteristics & Median (IQR) \\
\hline Age, $\mathrm{y}$ & $65.00(55.00,72.25)$ \\
Sex, $\mathrm{n}(\%)$ & $50(55.6)$ \\
Female & $40(44.4)$ \\
Male & \\
Severe, $\mathrm{n}(\%)$ & $16(17.8)$ \\
Yes & $71(78.9)$ \\
No & $38 / 45(84.4)$ \\
Fever, $\mathrm{n} / \mathrm{N}(\%)$ & $42 / 45(93.3)$ \\
Cough, $\mathrm{n} / \mathrm{N}(\%)$ & $34 / 45(75.6)$ \\
Dyspnea, $\mathrm{n} / \mathrm{N}(\%)$ & $28 / 45(62.2)$ \\
Headache, $\mathrm{n} / \mathrm{N}(\%)$ & $23 / 45(51.1)$ \\
Diarrhea, $\mathrm{n} / \mathrm{N}(\%)$ & $5.87(4.61,7.34)$ \\
Leukocyte count, $\times 10^{9} / \mathrm{L}$ & $3.85(2.65,4.80)$ \\
Neutrophil count, $\times 10^{9} / \mathrm{L}$ & $1.24(0.87,1.69)$ \\
Lymphocyte count, $\times 10^{9} / \mathrm{L}$ & $125.00(114.00,138.00)$ \\
Hemoglobin, g/L & $15.60(3.15,62.90)$ \\
CRP, mg/L & $23.50(14.75,42.25)$ \\
ALT, U/L & $23.50(18.00,37.00)$ \\
AST, U/L & $9.90(8.50,13.50)$ \\
Total bilirubin, $\mu \mathrm{mol} / \mathrm{L}$ &
\end{tabular}

CRP, C-reactive protein; ALT, Alanine aminotransferase; AST, Aspartate aminotransferase. 
Table 2 The comparison of IgM and IgG titers at week 4 and 6 [Median (IQR)]

\begin{tabular}{ccccc}
\hline & $\mathbf{4 w}$ & $\mathbf{6 w}$ & $\boldsymbol{Z}$ & $\boldsymbol{P}$ \\
\hline $\mathrm{IgM}, \mathrm{AU} / \mathrm{ml}$ & $60.50(26.50,115.50)$ & $17.50(7.64,39.00)$ & -7.551 & $<0.001$ \\
\hline $\mathrm{IgG}, \mathrm{AU} / \mathrm{ml}$ & $189.00(161.75,297.00)$ & $108.00(86.25,169.00)$ & -7.207 & $<0.001$ \\
\hline
\end{tabular}

Table 3 The comparison of IgM positive rate at week 4 and 6 [n(\%)]

\begin{tabular}{ccccc}
\hline & \multicolumn{2}{c}{ IgM-4w } & \multirow{2}{*}{$\chi^{2}$} & \multirow{2}{*}{$\boldsymbol{P}$} \\
\cline { 2 - 4 } IgM-6w & Negative & Positive & 17.048 & $<0.001$ \\
Negative & $6(100.0)$ & $21(26.9)$ & & \\
Positive & $0(0.0)$ & $57(73.1)$ & & \\
\hline
\end{tabular}

tive rate of $\operatorname{IgM}$ at week 4 and 6.-The positive rates of $\operatorname{IgM}$ at week 4 and 6 were $92.9 \%$ and $67.9 \%$, respectively. We found the difference in the positive rate of IgM between week 4 and 6 $(P<0.001)$. There are no differences in the positive rate of $\mathrm{IgG}$ between week 4 and 6 . The positive rates of $\operatorname{IgG}$ at week 4 and week 6 were $100 \%$. (Table 4). In order to observe the association of the positive rate of antibody with the severity of COVID-19, the patients were divided according to the severity of COVID-19. No association was observed in the positive rate of

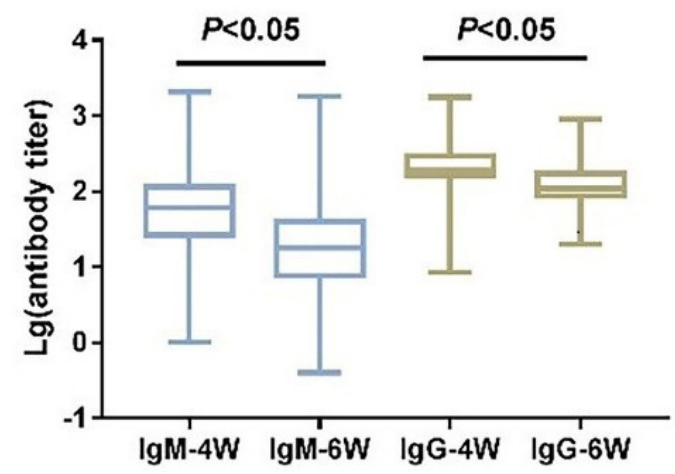

Fig. 1. The titers of IgM and IgG antibody in each patient antibody between severe patients and non-severe patients $(P>0.05)$ (Table 5).

In this study, the positive rate of nucleic acid test was $46.7 \%$, the negative rate was $53.3 \%$. No association was found between the positive rate of antibody detection at week 4 or 6 and that of nucleic acid test $(P>0.05)$ (Table 6).

\section{Discussion}

COVID-19 pneumonia has been confirmed as a risk of human-to-human transmission (5). It is necessary to find an accurate and rapid laboratory method for diagnosis. Nowadays, the serological test has become an approach to diagnose COVID-19. Data from the SARS outbreak indicate that viral antibodies are effective for serologic diagnosis (6). In addition, an epidemiological investigation of viral-specific IgM and IgG levels in the population will help to fully understand how many people have been infected. This information can determine the proportion of different types of infections and analyze the complete spectrum of COVID-19 (7). In our study, we assessed the responses of viral-specif-

Table 4 The comparison of IgG positive rates at week 4 and 6 [n(\%)]

\begin{tabular}{ccccc}
\hline & \multicolumn{2}{c}{ IgG-4w } & \multirow{2}{*}{$\chi^{2}$} & \multirow{2}{*}{} \\
\cline { 2 - 3 } & Negative & Positive & 0.000 & 1.000 \\
IgG-6w & & & & \\
Negative & $0(0.00)$ & $0(0.0)$ & & \\
Positive & $0(100.0)$ & $84(100.0)$ & & \\
\hline
\end{tabular}


ic IgM and $\mathrm{IgG}$ during SARS-CoV-2 infection and found no association of antibody detection with nucleic acid test in the positive rate. Most of infection cases in this study were the elderly, which is similar to other studies (8). Compared with young and middle-aged patients, elderly people with comorbidities are susceptible to SARS CoV-2 infection and are prone to develop severe illness, resulting in a higher mortality rate $(2,9)$. A percentage of 17.8 was represented by severely affected cases by the virus and $75.1 \%$ cases was not severely affected. The severity and case fatality rate of COVID-19 pneumonia are much lower than SARS and MERS. There is a certain relationship with the strict prevention and control efforts of the country. Close contacts need to be isolated and suspected infected persons are required to go to the designated hospital as soon as possible. Fever and cough were the common symptoms, which is consistent with recent studies $(10,11)$.

Zhang et al. (12) found that the positive rates of $\operatorname{IgM}$ and $\operatorname{IgG}$ were $81 \%$ and $100 \%$ on day 5 , respectively. The positive rate of IgG was similar to our result, but the positive rate of $\operatorname{IgM}$ was higher than our result. The possible reasons for

Table 5 The association between the positive rates of antibodies and severity of COVID-19 [n(\%)]

\begin{tabular}{|c|c|c|c|c|}
\hline & Severe & Non-severe & $\chi^{2}$ & $P$ \\
\hline IgM-4w & & & $<0.001$ & $>0.05$ \\
\hline Negative & $2(12.5)$ & $7(9.9)$ & & \\
\hline Positive & $14(87.5)$ & $64(90.1)$ & & \\
\hline IgG-4w & & & - & $>0.05^{*}$ \\
\hline Negative & $0(0.0)$ & $1(1.4)$ & & \\
\hline Positive & $16(100.0)$ & 70 (98.6) & & \\
\hline IgM-6w & & & $<0.001$ & 0.997 \\
\hline Negative & $5(35.7)$ & $21(31.3)$ & & \\
\hline Positive & $9(64.3)$ & $46(68.7)$ & & \\
\hline IgG-6w & & & - & - \\
\hline Negative & $0(0.0)$ & $0(0.0)$ & & \\
\hline Positive & $14(100.0)$ & $67(100.0)$ & & \\
\hline
\end{tabular}

*Fisher's exact test

Table 6 The association between antibody detection and nucleic acid test [n(\%)]

\begin{tabular}{|c|c|c|c|c|}
\hline \multirow{2}{*}{ Antibody detection } & \multicolumn{2}{|c|}{ Nucleic acid test } & \multirow[b]{2}{*}{$\chi^{2}$} & \multirow{2}{*}{$\mathbf{P}$} \\
\hline & Negative & Positive & & \\
\hline IgM-4w & & & - & $>0.05^{*}$ \\
\hline Negative & $5(10.4)$ & $4(9.5)$ & & \\
\hline Positive & $43(89.6)$ & $38(90.5)$ & & \\
\hline IgG-4w & & & - & $>0.05^{*}$ \\
\hline Negative & $1(2.1)$ & $0(0.0)$ & & \\
\hline Positive & $47(97.9)$ & $42(100.0)$ & & \\
\hline IgM-6w & & & 0.471 & 0.493 \\
\hline Negative & $13(28.9)$ & $14(35.9)$ & & \\
\hline Positive & $32(71.1)$ & $25(64.1)$ & & \\
\hline IgG-6w & & & - & - \\
\hline Negative & $0(0.0)$ & $0(0.0)$ & & \\
\hline Positive & $45(100.0)$ & $39(100.0)$ & & \\
\hline
\end{tabular}

*Fisher's exact test 
the difference are the different testing time, study population, and sample size. IgM can be detected 3-6 days after infection, the positive rate of antibody increased in time and then decreased. The study conducted by Du et al. (7) showed that antibody titers were consistent with clinical manifestations. They suggested that antibody detection can be used as an indicator to judge the progression stage of COVID-19 and the antibody levels of patients in recovery period were not always maintained at a high level. Our result is consistent with a study conducted by Hou et al. (13), which found that the positive rates of IgM and/or IgG antibody in mild, severe, and critical patients were not significantly different. In our study, we did not find a relationship between serological test and molecular detection in positive rate. The window period refers to the period from virus infecting human to the time when the virus antibody can be detected in peripheral blood. IgM antibodies are produced early and provide the first line of humoral immune defense, but the concentration is low, the maintenance time is short, which is a diagnostic indicator of acute phase infection. Afterwards, IgG antibodies are initiated and indicate mid-late or previous infection (14). Compared with serological test, nucleic acid detection can detect cases early. A number of studies assessed the diagnostic value of antibody detection, and proved antibody detection was an effective tool of diagnosis for SARS-CoV-2 infection $(15,16)$. However, few studies compared the positive rate of nucleic acid detection and serological test.

There are several limitations of this study. Firstly, selection bias was inevitable in this retrospective study, and the conclusions should be extended with caution because of the small sample size. Secondly, false-negative and false-positive results of nucleic acid test and antibody detection may influence the discoveries. Thirdly, the $4^{\text {th }}$ week refers to the time from the onset of symptom to blood collection for serological testing.
The antibody kit was not enough when the patient was admitted to the hospital, so the unified starting point for antibody testing was selected as week 4. Finally, the patient failed to perform antibody testing after discharge from the hospital to clarify the regularity of antibodies and the duration of maintenance in the body.

In conclusion, antibody detection is an effective means in the diagnosis of COVID-19. The titer and positive rate of IgM are lower than those of IgG. No association was found in positive rate between antibody detection and nucleic acid test.

\section{Abbreviation}

COVID-19: coronavirus disease 2019

SARS-CoV-2: severe acute respiratory syndrome coronavirus 2

RT-PCR: reverse transcription polymerase chain reaction

CT: computed tomography

CLIA: chemiluminescence immunoassay

SD: standard deviation

IQR: interquartile range

CRP: C-reactive protein;

ALT: Alanine aminotransferase

AST: Aspartate aminotransferase

\section{Acknowledgments}

Not applicable.

\section{Author Contributions}

JN, YQ, SYY, and SHY conceived and designed the protocol. YB, TJ, SH, and WS performed data collection and analysis. SYY and SHY contributed to manuscript preparation. All authors read and approved the final manuscript.

\section{Conflict of Interests}

The authors declare that there is no conflict of interests to disclose. 


\section{References}

1. Baud D, Qi X, Nielsen-Saines K, Musso D, Pomar L, Favre G. Real estimates of mortality following COVID-19 infection. Lancet Infect Dis. 2020 Jul;20(7):773. DOI: 10.1016/S1473-3099(20)30195-X

2. Huang C, Wang Y, Li X, Ren L, Zhao J, Hu Y, et al. Clinical features of patients infected with 2019 novel coronavirus in Wuhan, China. Lancet. 2020 Feb;395(10223):497-506. DOI: 10.1016/S01406736(20)30183-5

3. Lee HK, Lee BH, Seok SH, Baek MW, Lee HY, Kim DJ, et al. Production of specific antibodies against SARS-coronavirus nucleocapsid protein without cross reactivity with human coronaviruses $229 \mathrm{E}$ and OC43. J Vet Sci. 2010 Jun;11(2):165-7. DOI: 10.4142/ jvs.2010.11.2.165

4. National Health Commission of China and State Administration of Traditional Chinese Medicine. Notice on the issuance of strategic guidelines for diagnosis and treatment of novel coronavirus (2019-nCoV) infected pneumonia (seventh edition draft). 2020 Mar. http:// www.nhc.gov.cn/yzygj/s7653p/202003/46c9294a7dfe4cef80dc7f5912eb1989.shtml.

5. Chan JF, Yuan S, Kok KH, To KK, Chu H, Yang J, et al. A familial cluster of pneumonia associated with the 2019 novel coronavirus indicating person-to-person transmission: a study of a family cluster. Lancet. 2020 Feb;395(10223):514-23. DOI: 10.1016/S01406736(20)30154-9

6. Woo PC, Lau SK, Wong BH, Tsoi HW, Fung AM, Chan $\mathrm{KH}$, et al. Detection of specific antibodies to severe acute respiratory syndrome (SARS) coronavirus nucleocapsid protein for serodiagnosis of SARS coronavirus pneumonia. J Clin Microbiol. 2004 May;42(5):2306-9. DOI: 10.1128/JCM.42.5.2306-2309.2004

7. Du Z, Zhu F, Guo F, Yang B, Wang T. Detection of antibodies against SARS-CoV-2 in patients with COVID-19. J Med Virol. 2020 Apr;92(10):1735-8.
DOI: 10.1002/jmv.25820

8. Zhou S, Wang Y, Zhu T, Xia L. CT Features of Coronavirus Disease 2019 (COVID-19) Pneumonia in 62 Patients in Wuhan, China. AJR Am J Roentgenol. 2020 Jun;214(6):1287-94. DOI: 10.2214/AJR.20.22975

9. Wang $\mathrm{D}, \mathrm{Hu} \mathrm{B}, \mathrm{Hu} \mathrm{C}$, Zhu F, Liu X, Zhang J, et al. Clinical Characteristics of 138 Hospitalized Patients With 2019 Novel Coronavirus-Infected Pneumonia in Wuhan, China. Jama. 2020 Mar;323(11):1061-9. DOI: 10.1001/jama.2020.1585

10. Zhang JJ, Dong X, Cao YY, Yuan YD, Yang YB, Yan $Y Q$, et al. Clinical characteristics of 140 patients infected with SARS-CoV-2 in Wuhan, China. Allergy. 2020 Jul;75(7):1730-1741. DOI: 10.1111/all.14238

11. Zha L, Li S, Pan L, Tefsen B, Li Y, French N, et al. Corticosteroid treatment of patients with coronavirus disease 2019 (COVID-19). Med J Aust. 2020 May;212(9):41620. DOI: $10.5694 / \mathrm{mja} 2.50577$

12. Zhang W, Du RH, Li B, Zheng XS, Yang XL, Hu B, et al. Molecular and serological investigation of 2019-nCoV infected patients: implication of multiple shedding routes. Emerg Microbes Infect. 2020 Feb;9(1):386-9. DOI: 10.1080/22221751.2020.1729071

13. Hou H, Wang T, Zhang B, Luo Y, Mao L, Wang F, et al. Detection of IgM and IgG antibodies in patients with coronavirus disease 2019. Clin Transl Immunology. 2020 May;9(5):e01136. DOI: 10.1002/cti2.1136

14. Racine R, Winslow GM. IgM in microbial infections: taken for granted? Immunol Lett. 2009 Aug;125(2):7985. DOI: 10.1016/j.imlet.2009.06.003

15. Jin Y, Wang M, Zuo Z, Fan C, Ye F, Cai Z, et al. Diagnostic value and dynamic variance of serum antibody in coronavirus disease 2019. Int J Infect Dis. 2020 May;94:49-52. DOI: 10.1016/j.ijid.2020.03.065

16. Choe JY, Kim JW, Kwon HH, Hong HL, Jung CY, Jeon $\mathrm{CH}$, et al. Diagnostic performance of immunochromatography assay for rapid detection of $\operatorname{IgM}$ and $\mathrm{IgG}$ in coronavirus disease 2019. J Med Virol. 2020 May;92(11):2567-72. DOI: 10.1002/jmv.26060 